\title{
Intramolecular Through-Space Charge-Transfer Emitters Featuring Thermally Activated Delayed Fluorescence for High-Efficiency Electroluminescent Devices
}

\author{
Yongjun Song, Mingxing Tian, Renyou Yu, Lei He \\ Key Laboratory of Pesticide and Chemical Biology of Ministry of Education, Hubei \\ International Scientific and Technological Cooperation Base of Pesticide and Green \\ Synthesis, College of Chemistry, Central China Normal University, Wuhan 430079, \\ People's Republic of China. \\ E-mail:helei@mail.ccnu.edu.cn
}

\begin{abstract}
Through-space charge-transfer (TSCT) emitters have been extensively explored for thermally activated delayed fluorescence (TADF), but arranging various donors and acceptors into rigid cofacial conformations for various efficient TSCT TADF emitters has still remained a challenge. Here we report for the first time a "fixing acceptor" design to reach various efficient TSCT TADF emitters. By chemically fixing the acceptor (benzophenone) with a rigid spiro structure and cofacially aligning various donors with the fixed acceptor, a series of efficient TSCT TADF emitters have been developed. Single-crystal structures and theoretical calculations have verified closelypacked cofacial donor/acceptor conformations and favorable TSCT in the emitters. The emitters afford sky-blue to yellow TADF emission in doped films, with high photoluminescent efficiencies of up to 0.92 and reverse intersystem crossing rates of
\end{abstract}


up to $1.0 \times 10^{6} \mathrm{~s}^{-1}$. Organic light-emitting diodes using the emitters afford sky-blue to yellow electroluminescence with high external quantum efficiencies of up to $20.9 \%$. The work opens a new avenue toward a wide variety of efficient TSCT TADF emitters. Keywords: donor-acceptor systems, charge transfer, through space, thermally activated delayed fluorescence, electroluminescence

\section{Introduction}

Charge-transfer emitters with thermally activated delayed fluorescence (TADF) have aroused huge research interest for their wide applications in light-emitting devices [1-3], photocatalysis [4], and biological imaging [5], et al. They exhibit fast reverse intersystem crossing (RISC) from non-emissive triplet states $\left(T_{1}\right)$ to emissive singlet states $\left(\mathrm{S}_{1}\right)$, due to the small singlet-triplet energy gaps $\left(\Delta E_{\mathrm{ST}}\right)(<0.2 \mathrm{eV})$ produced by spatial separation of the highest occupied molecular orbitals (HOMOs) and the lowest unoccupied molecular orbitals (LUMOs) [1,6]. With advantages of triplet-harvesting and low-cost, they have demonstrated a bright prospect for the use in electroluminescence (EL) devices [2, 3].

While through-bond charge-transfer TADF emitters have been widely developed by directly linking donor (D) and acceptor (A) units via chemical bonds or conjugated $\pi$-linkers and carefully twisting the D/A conformations $[2,3]$, through-space chargetransfer (TSCT) TADF emitters have recently attracted fast-growing interest [7]. In the simplest manner, TSCT systems can be developed by physically blending D and A molecules $[6,8,9]$. Such TSCT systems (also known as exciplex systems) can afford 
tiny $\Delta E$ ST values $(\sim \mathrm{meV})$ due to the almost complete spatial separation of HOMO and LUMO orbitals, which are highly favorable for TADF [6, 10-15]. However, they usually afford low luminescent efficiencies, due to weak TSCT and pronounced non-radiative decay losses, resulting from loose packing and random orientations of the D/A molecules $[16,17]$. To solve this challenge, the D/A molecules have been held close by non-conjugated $\sigma$-linkers [18-24]. Nevertheless, these D- $\sigma$-A TSCT systems still show relatively low luminescent efficiencies because of the lack of control over the relative orientations of the D and A units.

Recent researches have proved that cofacial D/A alignments are favored for developing efficient TSCT emitters [25-38], which can reinforce TSCT and promote radiative decay of charge-transfer states [35, 37]. Very recently, Jiang and Liao et al. have unveiled that fixation of the cofacial D/A conformation is critically important for developing efficient TSCT emitters, which can remarkably suppresses non-radiative decay of charge-transfer states by restricting the rotation or vibration of the $\mathrm{D} / \mathrm{A}$ units [39]. The fixation has been achieved by chemically fixing the 9,10-dihydroacridine type D units and confining the D/A units into rigid cofacial conformations [39-46]. By this design, a series of highly efficient TSCT TADF emitters have been developed, which have proved high performances in EL devices [39-46]. Unfortunately, in this design, the $\mathrm{D}$ units are confined to be derivatives of 9,10-dihydroacridine [39-46], which imposes some restrictions on donor variation and further molecular engineering. It has still remained challenging to develop various efficient TSCT TADF emitters.

Herein, we report for the first time a "fixing acceptor" design to reach various 
efficient TSCT TADF emitters. As shown in Scheme 1, TSCT emitters 1-5 are developed by chemically fixing the A unit (benzophenone) and confining the D/A units into closely-packed cofacial conformations. The A unit is fixed with a spiro-structure derived from a carbonyl group of the A precursor. The D units are facilely varied with different structures and electron-donating strengths, which are 1,3,6,8-tetramethyl-9Hcarbazole (Me $4 \mathrm{CAZ}$ ), 9,9-dimethyl-9,10-dihydroacridine (DMAC), diphenylamine (DPA), triphenylamine (TPA), and benzo[5,6][1,4] oxazino[2,3,4-k1]phenoxazine (BOP) for 1-5, respectively. Single-crystal $X$-ray structures and theoretical calculations have verified closely-packed cofacial D/A conformations and favorable TSCT in 1-5. In doped films, 1-5 afford strong sky-blue to yellow TADF emission, with high photoluminescent efficiencies $\left(\Phi_{\mathrm{PL}}\right)$ of up to 0.92 and RISC rates $\left(k_{\mathrm{RISC}}\right)$ of up to $1.0 \times 10^{6} \mathrm{~s}^{-1}$. Organic light-emitting diodes (OLEDs) using 1-5 as the emitters show skyblue to yellow EL with high external quantum efficiencies (EQES) of up to $20.9 \%$.

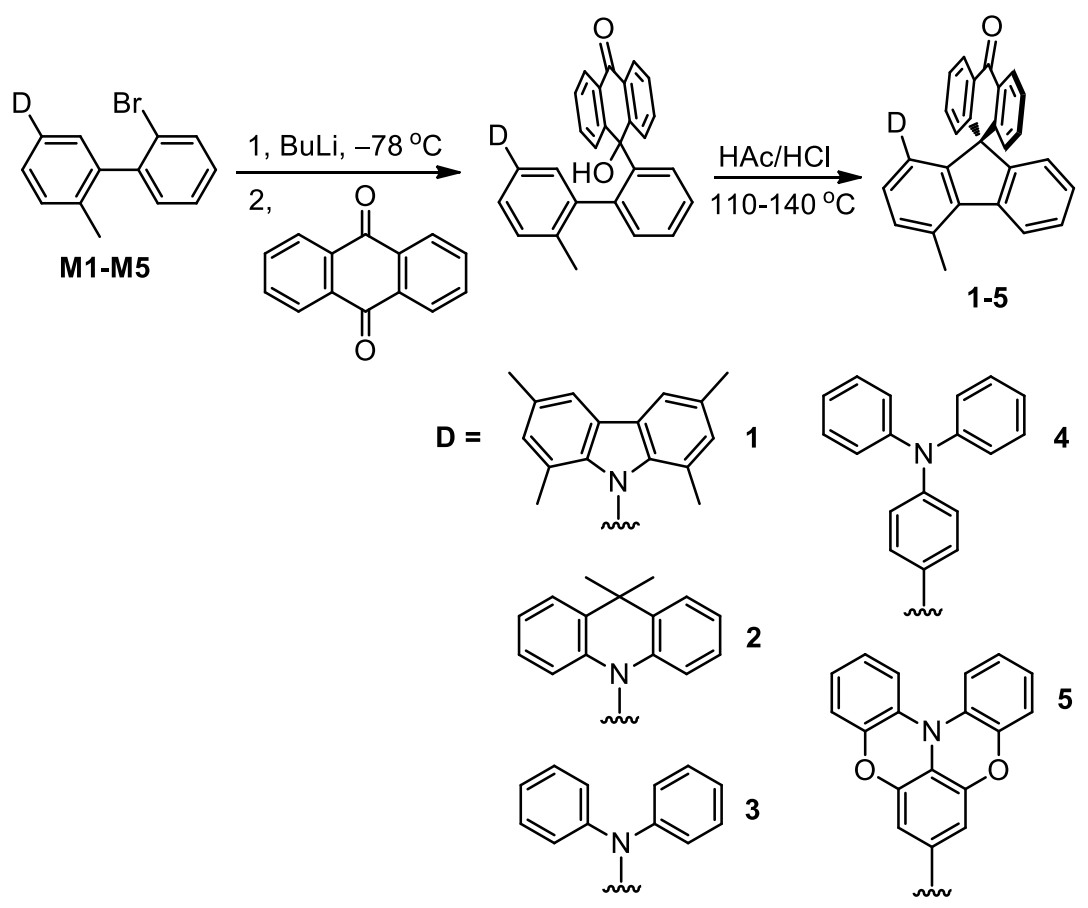

Scheme 1. Synthetic routes to TSCT emitters 1-5. 


\section{Results and discussion}

Scheme 1 outlines the general synthetic route to $1-5$. The rigid spiro-structure in a fluorene linker is utilized to fix benzophenone as the A unit. The D-containing compounds M1-M5 firstly reacted with $n$-butyllithium to form the phenyllithium reagents, which then reacted with one carbonyl group of the A precursor (anthraquinone) to afford the tertiary alcohol intermediates including both $\mathrm{D}$ and $\mathrm{A}$ units. By intramolecular Friedel-crafts cyclization reactions in the tertiary alcohol intermediates, 1-5 were prepared with total yields of $40-64 \%$. A key design is to direct the cyclization to occur at the ortho-position of the D unit by blocking the other cyclization site with a methyl group. Scheme S1 outlines the synthetic routes to the key intermediate compounds M1-M5. M3 was prepared by a $\mathrm{Cu}(\mathrm{I})$ catalyzed Ullman reaction between 2'-bromo-6-methyl-[1,1'-biphenyl]-3-amine (S1) and aniline, while M1, M2, M4 and M5 were synthesized by $\mathrm{Pd}(\mathrm{II})$ catalyzed Buchwald-Hartwig or Suzuki reactions between 2'-bromo-5-iodo-2-methyl-1,1'-biphenyl (S2) and D units or boronic acid derivatives of D units. Detailed synthesis procedures to S1, S2, M1-M5 and 1-5 are presented in Supporting Information. It is worth mentioning that the synthetic route reported here can apply to not only various D units but also different A precursors that carry a carbonyl group serving as the spiro-fixing site. 

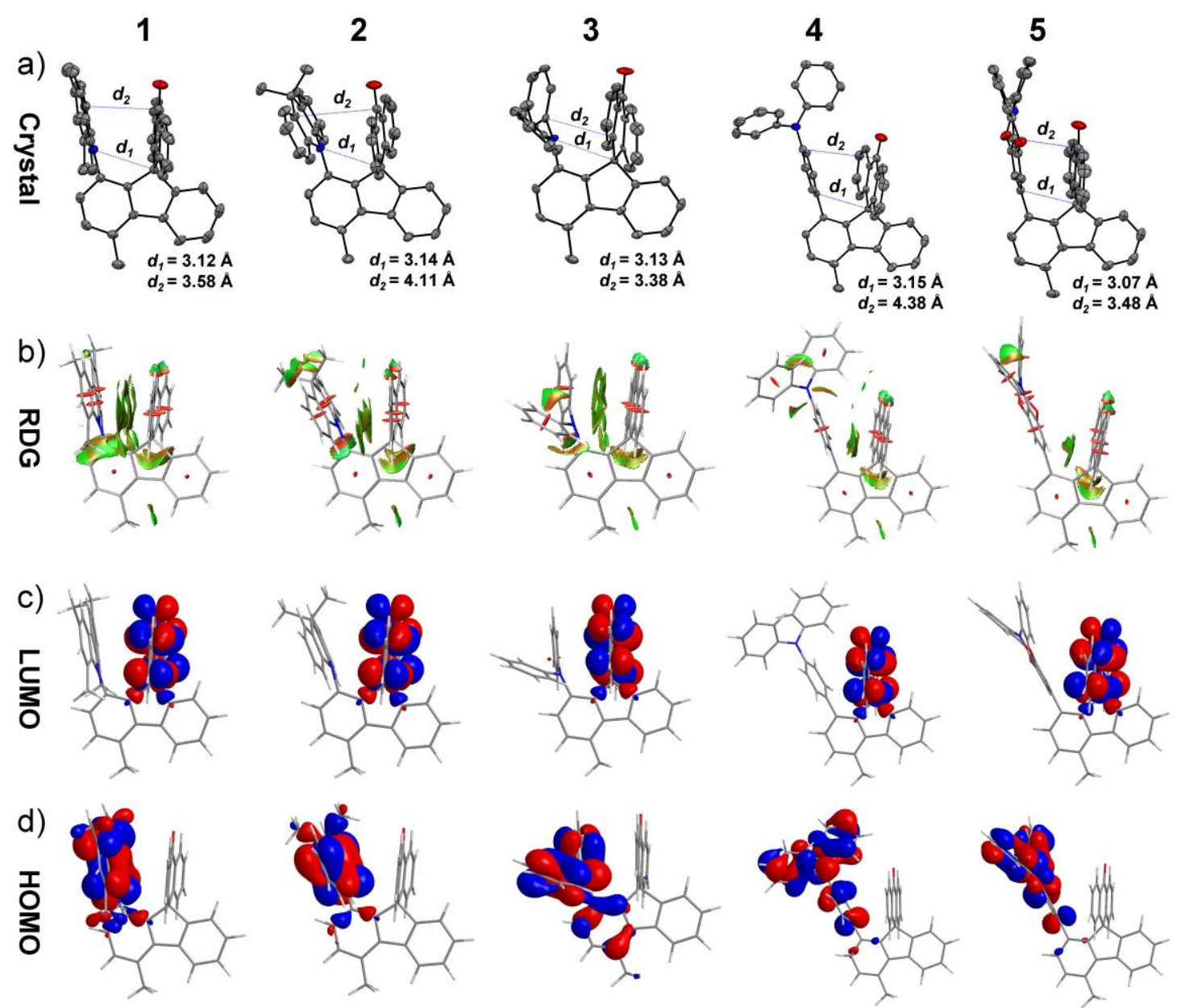

Figure 1. a) Single-crystal structures. b) Reduced density gradient (RDG) isosurfaces,

c) LUMO and d) HOMO plots on the optimized $\mathrm{S}_{0}$ states.

Figure 1a depicts the single-crystal $X$-ray structures of 1-5. The crystallographic data are summarized in Table S1. The D units and the spiro-fixed A units in 1-5 all display closely-packed cofacial configurations. The shortest distances between the nonhydrogen atoms of the $\mathrm{D}$ and $\mathrm{A}$ units are as short as $3.07-3.15 \AA$, indicating close $\mathrm{D} / \mathrm{A}$ interactions. Figure S1 illustrates intramolecular $\pi-\pi$ stacking interactions. For $1-5$, the $\mathrm{D}$ and $\mathrm{A}$ units stack to each other with the centroid-to-centroid distances and dihedral angles of the two stacking rings at 3.3-3.8 $\AA$ and $10-29^{\circ}$ (Figure S1 and Table S2), respectively, which indicates strong or considerable face-to-face $\pi-\pi$ stacking 
interactions [35, 39].

Theoretical calculations were conducted with density functional theory (DFT) (See ESI). Figure $1 b-d$ show the calculation results of the ground-states $\left(\mathrm{S}_{0}\right)$. On the optimized $\mathrm{S}_{0}$ states, the closely-packed D/A units exhibit considerable non-covalent van der Waals interactions, as revealed by the reduced density gradient (RDG) isosurfaces (Figure $1 \mathrm{~b}$ and Figure S2), which agree with the $\pi-\pi$ stacking interactions between the $\mathrm{D}$ and A units $[35,39]$. The HOMOs of 1, 2, 4 and 5 distribute predominantly over the D units, while the HOMO of 3 distributes over the diphenylamine unit, extending to the connected phenyl ring of the fluorene linker (Figure 1c). The LUMOs of 1-5 are all concentrated on the spiro-fixed benzophenone units (Figure 1d), with nearly no distributions on the fluorene linkers, due to the interrupted conjugation by the spirocarbons. It is shown that the HOMOs and LUMOs of 1-5 exhibit nearly no overlaps on the fluorene linkers, which minimizes through-bond charge-transfer. In contrast, the HOMOs and LUMOs largely overlap through the space due to the close cofacial D/A packing, which facilitates TSCT [35, 39]. Figure S3 depicts the natural transition orbital (NTO) analysis based on time-dependent DFT (TDDFT) calculations. For the $\mathrm{S}_{0} \rightarrow \mathrm{S}_{1}$ and $\mathrm{S}_{0} \rightarrow \mathrm{T}_{1}$ transitions, the hole and particle orbitals are predominantly delocalized over the D units and the spiro-fixed benzophenone units, respectively, which confirms the TSCT character of the $\mathrm{S}_{1}$ and $\mathrm{T}_{1}$ states. The calculated $\Delta E_{\mathrm{ST}}$ values of $1-5$ are as small as 8-27 meV (Table S3), which agrees with the almost complete spatial separation of HOMOs and LUMOs. The $\mathrm{S}_{1}$ and $\mathrm{T}_{1}$ states were further optimized with the TDDFT method. The relaxed $\mathrm{S}_{1}$ and $\mathrm{T}_{1}$ geometries show better cofacial $\mathrm{D} / \mathrm{A}$ 
alignments than the $\mathrm{S}_{0}$ geometries (Figure $\mathrm{S} 4$ ), as revealed by the shorter distances and more parallel alignments (except 3) between the D and A units (Table S2), due to the Coulomb attractive interactions on excited-states [39].

Figure S5 shows the absorption and photoluminescence (PL) spectra of 1-5 in toluene solution. Table S4 summarizes the photophysical characteristics. In addition to the strong absorption bands (below $350 \mathrm{~nm}$ for 1-4 and below $400 \mathrm{~nm}$ for 5) that arise from the D/A-centered ${ }^{1} \pi-\pi^{*} /{ }^{1} n-\pi^{*}$ transitions, 1-5 exhibit weak charge-transfer absorption bands at around $380 \mathrm{~nm}$ for $1-4$ and $410 \mathrm{~nm}$ for 5 (Figure S5a). The emergence of charge-transfer absorption indicates strong electronic coupling between the $\mathrm{D}$ and $\mathrm{A}$ units on ground states, due to the marked D/A overlap [39, 40, 45]. In the toluene solution, 1-5 afford green to orange emission centered at 514, 548, 524, 522 and $571 \mathrm{~nm}$ (Figure S5b), respectively. The different emission energies for 1-5 result from the different electron-donating strengths of the D units, as shown by electrochemical measurements (vide infra). The gradual red-shift of the emission by increasing the solvent polarity agrees with the charge-transfer character of the emission (Figure S6). In degassed toluene solution, 1-5 afford $\Phi_{\mathrm{PL}}$ below 0.10 (Table S4), which suggests considerable non-radiative decay losses in the fluid solution, presumably caused by vibrations or rotations of the unfixed D units (especially for 3 and 4 with rotary phenyl rings) $[29,43]$. 1-3 show both prompt and delayed components in the transient PL decays (Figure S7 and Table S4), reflecting the TADF feature of the emission, whereas 4 and 5 do not show delayed components, presumably because the $\mathrm{T}_{1}$ states are mostly consumed by non-radiative deactivations before being converted 
to the $\mathrm{S}_{1}$ states. 1-5 all display aggregation-induced emission phenomena, as revealed by the remarkable enhancement of PL intensity upon increasing the water fraction in the mixed $\mathrm{THF} / \mathrm{H}_{2} \mathrm{O}$ solution (Figure $\mathrm{S} 8$ ), due to suppressed vibrations or rotations of molecular fragments in aggregation states [24, 25].
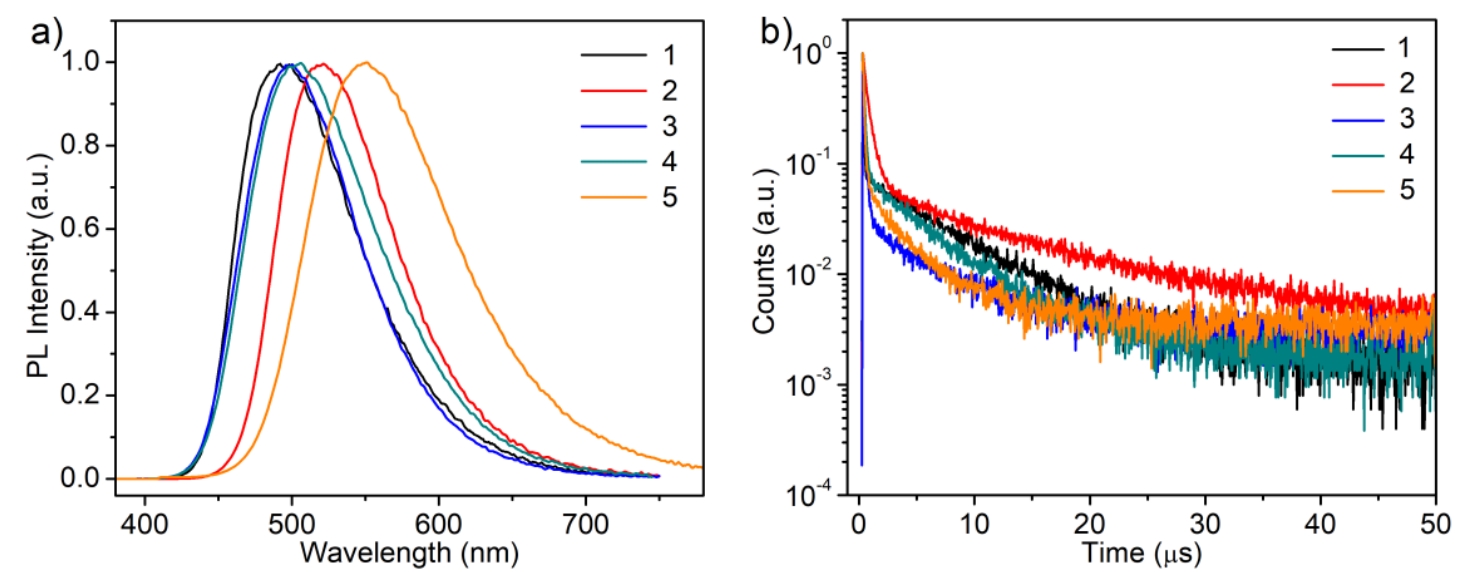

Figure 2. a) PL spectra and b) transient PL decay curves of $1-5$ in $30 \mathrm{wt} . \%$ doped DPEPO films.

Table 1. Summary of emission characteristics and excited-state energy levels of 1-5 in 30 wt.\% doped DPEPO films.

\begin{tabular}{cccccc}
\hline & $\mathrm{PL} \lambda(\mathrm{nm})$ & $\phi_{\mathrm{PL}}$ & $\tau_{\mathrm{p}}(\mathrm{ns})$ & $\tau_{\mathrm{d}}(\mu \mathrm{s})$ & $E_{\mathrm{S}} / E_{\mathrm{T}} / \Delta E_{\mathrm{ST}}(\mathrm{eV})$ \\
\hline 1 & 493 & 0.92 & 64 & 6.8 & $2.79 / 2.76 / 0.02$ \\
2 & 520 & 0.80 & 457 & 10.9 & $2.67 / 2.60 / 0.07$ \\
3 & 497 & 0.82 & 130 & 5.9 & $2.77 / 2.76 / 0.01$ \\
4 & 504 & 0.89 & 132 & 5.3 & $2.79 / 2.76 / 0.03$ \\
5 & 550 & 0.52 & 99 & 3.3 & $2.61 / 2.58 / 0.03$ \\
\hline
\end{tabular}

Figure 2a shows the PL spectra of $1-5$ in 30 wt.\% doped bis[2(diphenylphosphino)phenyl] ether oxide (DPEPO) films. Table 1 summarizes the 
emission characteristics. In the doped films, 1-5 afford sky-blue to yellow chargetransfer emission centered at 493, 520, 497, 504 and $550 \mathrm{~nm}$, respectively, with high $\Phi_{\mathrm{PL}}$ values of $0.80-0.92$ for $1-4$ and a moderate $\Phi_{\mathrm{PL}}$ of 0.52 for 5 (Table 1 ). The remarkable enhancement of $\Phi_{\mathrm{PL}}$ in doped films in comparison with the solution results from further suppression of intramolecular vibrations/rotations in the solid state [35, 39]. Figure S9 shows the low-temperature fluorescence and phosphorescence spectra of the doped films. From the onsets of the fluorescence and phosphorescence spectra, singlet $\left(E_{\mathrm{S}}\right)$ and triplet $\left(E_{\mathrm{T}}\right)$ energies were respectively calculated (Table 1). 1-5 afford $\Delta E_{\mathrm{ST}}$ values at $10-70 \mathrm{meV}$, which are sufficiently small for rapid RISC. Figure $2 \mathrm{~b}$ shows the transient PL decay curves. 1-5 all show distinct prompt and delayed components in the transient PL decays, with prompt $\left(\tau_{\mathrm{p}}\right) /$ delayed $\left(\tau_{\mathrm{d}}\right)$ lifetimes at 64 ns/6.8 $\mu \mathrm{s}, 457 \mathrm{~ns} / 10.9 \mu \mathrm{s}, 130 \mathrm{~ns} / 5.9 \mu \mathrm{s}, 132 \mathrm{~ns} / 5.3 \mu \mathrm{s}$ and $99 \mathrm{~ns} / 3.3 \mu \mathrm{s}$, respectively. The delayed components are remarkably suppressed at low temperature (Figure S10), which confirms the TADF nature of 1-5. Table S5 summarizes the calculated rate constants of the emission in the doped films. 1-5 show radiative decay rates of singlet state $\left(k_{r, s}\right)$ at $0.7-3.3 \times 10^{6} \mathrm{~s}^{-1}$, which are within the normal range reported for TSCT emitters $[35,37,39-43,45] .1-4$ show non-radiative decay rates of singlet state $\left(k_{\mathrm{nr}, \mathrm{s}}\right)$ at $1.8-7.3 \times 10^{5} \mathrm{~s}^{-1}$, whereas yellow-emitting 5 exhibits a much larger $k_{\mathrm{nr}, \mathrm{s}}$ of $2.0 \times 10^{6} \mathrm{~s}^{-1}$, in accordance with the energy gap law. Notably, $1-5$ exhibit high $k$ RISC values at $9.9 \times 10^{5}$ $\mathrm{s}^{-1}, 2.3 \times 10^{5} \mathrm{~s}^{-1}, 3.3 \times 10^{5} \mathrm{~s}^{-1}, 6.1 \times 10^{5} \mathrm{~s}^{-1}$ and $7.1 \times 10^{5} \mathrm{~s}^{-1}$, respectively, indicating their high capability of converting triplets to singlets for emission.

Figure S11 shows the cyclic voltammograms measured in solution. 1-5 show 
similar electrochemical reductions and LUMO levels (around $-2.70 \mathrm{eV}$ ) but different electrochemical oxidations and HOMO levels $(-5.23 \sim-4.99 \mathrm{eV})$ (Table S6), because they use the same A unit and different D units. The Me4CAZ, DPA and TPA donors have similar electron-donating strengths, which lead to similar HOMO levels (around $-5.2 \mathrm{eV}$ ) and emission energies for 1, 3 and 4 (Figure 2a). The DMAC and BOP donors have increased electron-donating strengths, which lead to destabilized HOMO levels $(-5.14$ and $-4.99 \mathrm{eV}$, respectively) and decreased emission energies for 2 and 5 (Figure 2a). As shown by thermal gravity analysis (Figure S12), 1-5 all possess high thermal stability, with $5 \%$ weight loss temperatures at $401,385,384,417$ and $444{ }^{\circ} \mathrm{C}$, respectively.
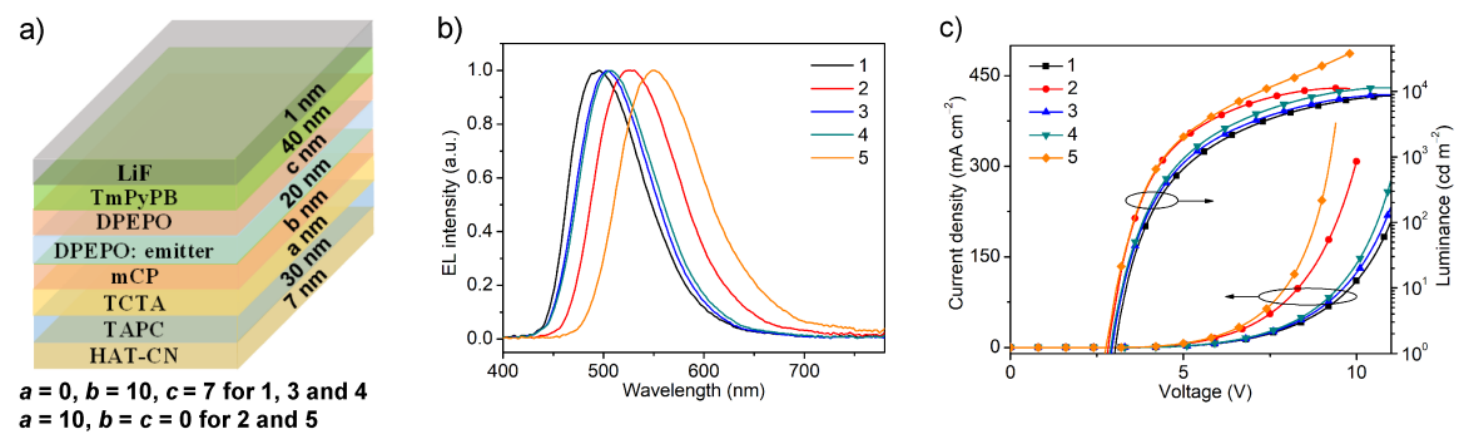

Figure 3. a) Device structures, b) EL spectra and c) current-density and luminance versus driving voltage curves of OLEDs using 1-5 as the emitters.

To evaluate their application potential in EL devices, 1-5 were employed as emitters in vacuum-evaporated OLEDs. Figure 3a illustrates the device structures, which are indium-tin-oxide (ITO)/HATCN (7 nm)/TAPC (30 nm)/mCP (10 nm)/PPF: 30 wt.\% emitter $(20 \mathrm{~nm}) / \mathrm{PPF}(7 \mathrm{~nm}) / \mathrm{TmPyPB}(40 \mathrm{~nm}) / \mathrm{LiF}(1 \mathrm{~nm}) / \mathrm{Al}(100 \mathrm{~nm})$ for 
sky-blue to blue-green-emitting 1,3 and 4 , and ITO/HATCN (7 nm)/TAPC (30 nm)/TCTA (10 nm)/PPF: 30 wt.\% emitter (20 nm)/TmPyPB (40 nm)/LiF (1 nm)/Al $(100 \mathrm{~nm})$ for green to yellow-emitting 2 and 5. Scheme S2 illustrates the energy-level diagrams of the devices as well as the chemical structures of the used materials. Here, 1,4,5,8,9,11-hexaazatriphenylenehexacarbonitrile (HAT-CN) and 1,1-bis[(di-4tolylamino)phenyl]cyclohexane (TAPC) function as the hole-injection and transport layers, respectively; 1,3-di(9H-carbazol-9-yl)benzene (mCP) or 4,4',4"'-tris-(9Hcarbazol-9-yl)-triphenylamine (TCTA) serve as the electron/exciton-blocking layer; 2,8-bis(diphenylphosphoryl)dibenzo[b,d]furan (PPF), an analog of DPEPO, serves as not only the host but also the hole/exciton-blocking layer; 1,3,5-tri[(3-pyridyl)-phen-3yl]benzene (TmPyPB) and $\mathrm{LiF}$ serve as the electron-transport and injection layers, respectively. Figure $3 \mathrm{~b}$ shows the EL spectra. The OLEDs based on 1-5 afforded skyblue to yellow EL with the emission peaks at 494, 527, 503, 507 and $550 \mathrm{~nm}$, respectively, and Commission Internationale de l'Eclairage (CIE) coordinates at (0.20, $0.42),(0.31,0.56),(0.22,0.48),(0.24,0.50)$ and $(0.41,0.55)$, respectively.

Table 2. Summary of EL performances of OLEDs using 1-5 as the emitters.

\begin{tabular}{ccccccc}
\hline & $\begin{array}{c}V_{\text {on }} \\
(\mathrm{V})^{a}\end{array}$ & $\begin{array}{c}\eta_{\mathrm{c}} \\
\left(\mathrm{cd} \mathrm{A}^{-1}\right)^{b}\end{array}$ & $\begin{array}{c}E Q E \\
(\%)^{c}\end{array}$ & $\begin{array}{c}L_{\max } \\
\left(\mathrm{cd} \mathrm{m}^{-2}\right)\end{array}$ & $\begin{array}{c}\text { EL } \\
\lambda(\mathrm{nm})\end{array}$ & $\begin{array}{c}\text { CIE } \\
(x, y)\end{array}$ \\
\hline 1 & 3.0 & $50.4 / 43.9 / 32.9$ & $20.9 / 18.2 / 13.7$ & 8600 & 494 & $(0.20,0.42)$ \\
2 & 2.8 & $52.4 / 52.0 / 43.9$ & $16.1 / 16.0 / 13.5$ & 11000 & 527 & $(0.31,0.56)$ \\
3 & 2.9 & $49.4 / 47.7 / 36.5$ & $17.7 / 17.3 / 13.3$ & 8800 & 503 & $(0.22,0.48)$ \\
4 & 2.9 & $56.3 / 54.2 / 43.9$ & $20.0 / 19.1 / 15.6$ & 11000 & 507 & $(0.24,0.50)$ \\
5 & 2.7 & $43.5 / 43.2 / 38.5$ & $13.2 / 13.1 / 11.7$ & 39000 & 550 & $(0.41,0.55)$ \\
\hline
\end{tabular}

${ }^{\mathrm{a}}$ Voltage to reach $1 \mathrm{~cd} \mathrm{~m}^{-2} .{ }^{\mathrm{b}}$ Current efficiencies at the maximum, $100 \mathrm{~cd} \mathrm{~m}^{-2}$ and 1000 
$\mathrm{cd} \mathrm{m}^{-2} \cdot{ }^{\mathrm{c}} E Q E \mathrm{~s}$ at the maximum, $100 \mathrm{~cd} \mathrm{~m}^{-2}$ and $1000 \mathrm{~cd} \mathrm{~m}^{-2}$.

Figure $3 \mathrm{c}$ shows the current-density and luminance versus voltage curves. Table 2 summarizes the device performances. The OLEDs based on 1-5 showed low turn-on voltages below $3.0 \mathrm{~V}$ and high peak current efficiencies/EQEs at $50.4 \mathrm{~cd} \mathrm{~A}^{-1} / 20.9 \%$, $52.4 \mathrm{~cd} \mathrm{~A} \mathrm{c}^{-1} / 16.1 \%, 49.4 \mathrm{~cd} \mathrm{~A}^{-1} / 17.7 \%, 56.3 \mathrm{~cd} \mathrm{~A}^{-1} / 20.0 \%$ and $43.5 \mathrm{~cd} \mathrm{~A}^{-1} / 13.2 \%$, respectively. The $E Q E$ s follow the trend of $\Phi_{\mathrm{PL}}$ in the doped films (Table 1). Figure S13 shows the EQE versus luminance curves. At $100 / 1000 \mathrm{~cd} \mathrm{~m}^{-2}$, the EQEs remained at $18.2 \% / 13.7 \%, \quad 16.0 \% / 13.5 \%, \quad 17.3 \% / 13.3 \%, 19.1 \% / 15.6 \%$ and $13.1 \% / 11.7 \%$ for devices based on 1-5, respectively. The devices based on 1, 3 and 4 showed relatively large efficiency roll-offs (Table 2). Efficiency roll-offs in TADF OLEDs are usually ascribed to triplet-triplet/triplet-polaron annihilations in the emissive layers [47]. The high $k_{\mathrm{RISC}}$ values of 1-5 indicate that they can efficiently consume triplets for emission, alleviating triplet-triplet/triplet-polaron annihilations [48-51]. By broadening the recombination zones with optimized device structures, the efficiency roll-offs would be suppressed $[35,36]$. The high device efficiencies indicate that $1-5$ are promising functional materials for high-performance EL devices.

\section{Conclusion}

We reported a "fixing acceptor" design for efficient TSCT TADF emitters. By chemically fixing the A unit (benzophenone) and cofacially aligning various D units with the fixed A unit, TSCT TADF emitters 1-5 were developed. Single-crystal 
structures and theoretical calculations verified closely-packed cofacial D/A conformations and favorable TSCT in 1-5. In doped films, 1-5 afforded high $\Phi_{\mathrm{PL}}$ of up to 0.92 and $k_{\text {RISC }}$ of up to $1.0 \times 10^{6} \mathrm{~s}^{-1}$. OLEDs using them as emitters showed high EQEs of up to $20.9 \%$. The work has demonstrated that fixing the acceptor can afford efficient TSCT TADF emitters. In principle, the "fixing acceptor" design allows facile variation of donor and feasible variation of acceptor as long as the acceptor precursors carry a carbonyl group that serves as the spiro-fixing site (such as thioxanthen-9-one sulfone and its derivatives). Varying the fixed acceptor is currently ongoing in our lab. The work opens a new avenue toward a wide variety of efficient TSCT TADF emitters, which are promising functional materials for high-performance EL devices.

\section{Acknowledgement}

We thank the National Natural Science Foundation of China (Grant No. 51773074), the Natural Science Foundation of Hubei Province (Grant No. 2020CFB762) and the program of introducing talents of discipline to universities of China (111 program, B17019) for financial support.

\section{Conflict of interest}

The authors declare no conflict of interest.

\section{References}

[1] H. Uoyama, K. Goushi, K. Shizu, H. Nomura, C. Adachi, Highly efficient organic lightemitting diodes from delayed fluorescence, Nature 492 (2012) 234-238. 
[2] M.Y. Wong, E. Zysman-Colman, Purely organic thermally activated delayed fluorescence materials for organic light-emitting diodes, Adv. Mater. 29 (2017) 1605444.

[3] Y. Liu, C. Li, Z. Ren, S. Yan, M.R. Bryce, All-organic thermally activated delayed fluorescence materials for organic light-emitting diodes, Nat. Rev. Mater. 3 (2018) 18020.

[4] M.A. Bryden, E. Zysman-Colman, Organic thermally activated delayed fluorescence (TADF) compounds used in photocatalysis, Chem. Soc. Rev. 50 (2021) 7587-7680.

[5] N. Van-Nghia, A. Kumar, M.H. Lee, J. Yoon, Recent advances in biomedical applications of organic fluorescence materials with reduced singlet-triplet energy gaps, Coordin. Chem. Rev. 425 (2020) 213545.

[6] K. Goushi, K. Yoshida, K. Sato, C. Adachi, Organic light-emitting diodes employing efficient reverse intersystem crossing for triplet-to-singlet state conversion, Nat. Photonics 6 (2012) 253-258.

[7] Q. Xue, G. Xie, Thermally activated delayed fluorescence beyond through-bond charge transfer for high-performance oleds, Adv. Opt. Mater. 9 (2021) 2002204.

[8] M. Sarma, K.-T. Wong, Exciplex: An intermolecular charge-transfer approach for TADF, ACS Appl. Mater. Interfaces 10 (2018) 19279-19304.

[9] M. Zhang, C.-J. Zheng, H. Lin, S.-L. Tao, Thermally activated delayed fluorescence exciplex emitters for high-performance organic light-emitting diodes, Mater. Horiz. 8 (2021) 401-425.

[10] X.-K. Liu, Z. Chen, C.-J. Zheng, C.-L. Liu, C.-S. Lee, F. Li, X.-M. Ou, X.-H. Zhang, Prediction and design of efficient exciplex emitters for high-efficiency, thermally activated delayed-fluorescence organic light-emitting diodes, Adv. Mater. 27 (2015) 2378-2383.

[11] M. Colella, A. Danos, A.P. Monkman, Less is more: Dilution enhances optical and electrical performance of a TADF exciplex, J. Phys. Chem. Lett. 10 (2019) 793-798.

[12] B. Liang, J. Wang, Z. Cheng, J. Wei, Y. Wang, Exciplex-based electroluminescence: Over $21 \%$ external quantum efficiency and approaching $100 \mathrm{~lm} / \mathrm{w}$ power efficiency, J. Phys. Chem. Lett. 10 (2019) 2811-2816.

[13] M. Chapran, P. Pander, M. Vasylieya, G. Wiosna-Salyga, J. Ulanski, F.B. Dias, P. Data, Realizing 20\% external quantum efficiency in electroluminescence with efficient thermally activated delayed fluorescence from an exciplex, ACS Appl. Mater. Interfaces 11 (2019) 13460-13471.

[14] C. Zhang, Y. Lu, Z. Liu, Y. Zhang, X. Wang, D. Zhang, L. Duan, A pi-D and pi-A exciplexforming host for high-efficiency and long-lifetime single-emissive-layer fluorescent white organic light-emitting diodes, Adv. Mater. 32 (2020) 2004040.

[15] J.H. Yun, K.H. Lee, W.J. Chung, J.Y. Lee, Y. Lee, J.J. Lyu, Thermally activated delayed fluorescence type exciplex host for long lifetime in deep blue phosphorescent organic lightemitting diodes, Chem. Eng. J. 417 (2021) 128086.

[16] K.-H. Kim, S.-J. Yoo, J.-J. Kim, Boosting triplet harvest by reducing nonradiative transition of exciplex toward fluorescent organic light-emitting diodes with $100 \%$ internal quantum efficiency, Chem. Mater. 28 (2016) 1936-1941.

[17] C.-K. Moon, J.-S. Huh, J.-M. Kim, J.-J. Kim, Electronic structure and emission process of excited charge transfer states in solids, Chem. Mater. 30 (2018) 5648-5654.

[18] G. Mehes, H. Nomura, Q. Zhang, T. Nakagawa, C. Adachi, Enhanced electroluminescence efficiency in a spiro-acridine derivative through thermally activated delayed fluorescence, 
Angew. Chem. Int. Edit. 51 (2012) 11311-11315.

[19] K. Kawasumi, T. Wu, T. Zhu, H.S. Chae, T. Van Voorhis, M.A. Baldo, T.M. Swager, Thermally activated delayed fluorescence materials based on homoconjugation effect of donor-acceptor triptycenes, J. Am. Chem. Soc. 137 (2015) 11908-11911.

[20] Y. Geng, A. D'Aleo, K. Inada, L.-S. Cui, J.U. Kim, H. Nakanotani, C. Adachi, Donorsigma-acceptor motifs: Thermally activated delayed fluorescence emitters with dual upconversion, Angew. Chem. Int. Edit. 56 (2017) 16536-16540.

[21] S. Shao, J. Hu, X. Wang, L. Wang, X. Jing, F. Wang, Blue thermally activated delayed fluorescence polymers with nonconjugated backbone and through-space charge transfer effect, J. Am. Chem. Soc. 139 (2017) 17739-17742.

[22] Y.-Z. Shi, K. Wang, X. Li, G.-L. Dai, W. Liu, K. Ke, M. Zhang, S.-L. Tao, C.-J. Zheng, X.M. Ou, X.-H. Zhang, Intermolecular charge-transfer transition emitter showing thermally activated delayed fluorescence for efficient non-doped oleds, Angew. Chem. Int. Edit. 57 (2018) 9480-9484.

[23] D.-D. Zhang, K. Suzuki, X.-Z. Song, Y. Wada, S. Kubo, L. Duan, H. Kaji, Thermally activated delayed fluorescent materials combining intra- and intermolecular charge transfers, ACS Appl. Mater. Interfaces 11 (2019) 7192-7198.

[24] J. Hu, Q. Li, X. Wang, S. Shao, L. Wang, X. Jing, F. Wang, Developing through-space charge transfer polymers as a general approach to realize full-color and white emission with thermally activated delayed fluorescence, Angew. Chem. Int. Edit. 58 (2019) 8405-8409.

[25] H. Tsujimoto, D.-G. Ha, G. Markopoulos, H.S. Chae, M.A. Baldo, T.M. Swager, Thermally activated delayed fluorescence and aggregation induced emission with through-space charge transfer, J. Am. Chem. Soc. 139 (2017) 4894-4900.

[26] E. Spuling, N. Sharma, I.D.W. Samuel, E. Zysman-Colman, S. Braese, (deep) blue through-space conjugated TADF emitters based on 2.2 paracyclophanes, Chem. Commun. 54 (2018) 9278-9281.

[27] M. Auffray, D.H. Kim, J.U. Kim, F. Bencheikh, D. Kreher, Q. Zhang, A. D'Aleo, J.-C. Ribierre, F. Mathevet, C. Adachi, Dithia 3.3 paracyclophane core: A versatile platform for triplet state fine-tuning and through-space TADF emission, Chem. Asian J. 14 (2019) 1921 1925.

[28] K.-L. Woon, C.-L. Yi, K.-C. Pan, M.K. Etherington, C.-C. Wu, K.-T. Wong, A.P. Monkman, Intramolecular dimerization quenching of delayed emission in asymmetric d-d '-a TADF emitters, J. Phys. Chem. C 123 (2019) 12400-12410.

[29] J.-A. Lin, S.-W. Li, Z.-Y. Liu, D.-G. Chen, C.-Y. Huang, Y.-C. Wei, Y.-Y. Chen, Z.-H. Tsai, C.-Y. Lo, W.-Y. Hung, K.-T. Wong, P.-T. Chou, Bending-type electron donor-donoracceptor triad: Dual excited-state charge-transfer coupled structural relaxation, Chem. Mater. 31 (2019) 5981-5992.

[30] X. Wang, S. Wang, J. Lv, S. Shao, L. Wang, X. Jing, F. Wang, Through-space charge transfer hexaarylbenzene dendrimers with thermally activated delayed fluorescence and aggregation-induced emission for efficient solution-processed oleds, Chem. Sci. 10 (2019) 2915-2923.

[31] Q. Li, J. Hu, J. Lv, X. Wang, S. Shao, L. Wang, X. Jing, F. Wang, Through-space chargetransfer polynorbornenes with fixed and controllable spatial alignment of donor and acceptor for high-efficiency blue thermally activated delayed fluorescence, Angew. Chem. 
Int. Edit. 59 (2020) 20174-20182.

[32] K. Li, Y. Zhu, B. Yao, Y. Chen, H. Deng, Q. Zhang, H. Zhan, Z. Xie, Y. Cheng, Rotationrestricted thermally activated delayed fluorescence compounds for efficient solutionprocessed oleds with eqes of up to $24.3 \%$ and small roll-off, Chem. Commun. 56 (2020) 5957-5960.

[33] Y. Wada, H. Nakagawa, S. Matsumoto, Y. Wakisaka, H. Kaji, Organic light emitters exhibiting very fast reverse intersystem crossing, Nat. Photonics 14 (2020) 643-649.

[34] X. Wang, J. Hu, J. Lv, Q. Yang, H. Tian, S. Shao, L. Wang, X. Jing, F. Wang, Pi-stacked donor-acceptor dendrimers for highly efficient white electroluminescence, Angew. Chem. Int. Edit. 60 (2021) 16585-16593.

[35] C. Wu, W. Liu, K. Li, G. Cheng, J. Xiong, T. Teng, C.-M. Che, C. Yang, Face-to-face orientation of quasiplanar donor and acceptor enables highly efficient intramolecular exciplex fluorescence, Angew. Chem. Int. Edit. 60 (2021) 3994-3998.

[36] Y.-P. Zhang, X. Liang, X.-F. Luo, S.-Q. Song, S. Li, Y. Wang, Z.-P. Mao, W.-Y. Xu, Y.-X. Zheng, J.-L. Zuo, Y. Pan, Chiral spiro-axis induced blue thermally activated delayed fluorescence material for efficient circularly polarized oleds with low efficiency roll-off, Angew. Chem. Int. Edit. 60 (2021) 8435-8440.

[37] L. He, R. Bai, R. Yu, X. Meng, M. Tian, X. Wang, Donor/acceptor pairs created by electrostatic interaction: Design, synthesis, and investigation on the exciplex formed within the pair, Angew. Chem. Int. Edit. 60 (2021) 6013-6020.

[38] S. Kumar, L.G. Franca, K. Stavrou, E. Crovini, D.B. Cordes, A.M.Z. Slawin, A.P. Monkman, E. Zysman-Colman, Investigation of intramolecular through-space chargetransfer states in donor-acceptor charge-transfer systems, J. Phys. Chem. Lett. 12 (2021) 2820-2830.

[39] X. Tang, L.S. Cui, H.C. Li, A.J. Gillett, F. Auras, Y.K. Qu, C. Zhong, S.T.E. Jones, Z.Q. Jiang, R.H. Friend, L.S. Liao, Highly efficient luminescence from space-confined chargetransfer emitters, Nat. Mater. 19 (2020) 1332-1338.

[40] C.-C. Peng, S.-Y. Yang, H.-C. Li, G.-H. Xie, L.-S. Cui, S.-N. Zou, C. Poriel, Z.-Q. Jiang, L.-S. Liao, Highly efficient thermally activated delayed fluorescence via an unconjugated donor-acceptor system realizing eqe of over 30\%, Adv. Mater. 32 (2020) 2003885.

[41] S.-Y. Yang, Y.-K. Wang, C.-C. Peng, Z.-G. Wu, S. Yuan, Y.-J. Yu, H. Li, T.-T. Wang, H.-C. Li, Y.-X. Zheng, Z.-Q. Jiang, L.-S. Liao, Circularly polarized thermally activated delayed fluorescence emitters in through-space charge transfer on asymmetric spiro skeletons, J. Am. Chem. Soc. 142 (2020) 17756-17765.

[42] T.-T. Wang, G. Xie, H.-C. Li, S.-Y. Yang, H. Li, Y.-L. Xiao, C. Zhong, K. Sarvendra, A. Khan, Z.-Q. Jiang, L.-S. Liao, П-stacked thermally activated delayed fluorescence emitters with alkyl chain modulation, CCS Chem. 2 (2020) 1757-1763.

[43] S.-Y. Yang, Q.L.S. Tian, Y.-J. Yu, S.-N. Zou, H.-C. Li, A. Khan, Q.-H. Wu, Z.-Q. Jiang, L.S. Liao, Sky-blue thermally activated delayed fluorescence with intramolecular spatial charge transfer based on a dibenzothiophene sulfone emitter, J. Org. Chem. 85 (2020) 10628-10637.

[44] H.-C. Li, X. Tang, S.-Y. Yang, Y.-K. Qu, Z.-Q. Jiang, L.-S. Liao, Spatial donor/acceptor architecture for intramolecular charge-transfer emitter, Chinese Chem. Lett. 32 (2021) 1245-1248. 
[45] X.-Q. Wang, S.-Y. Yang, Q.-S. Tian, C. Zhong, Y.-K. Qu, Y.-J. Yu, Z.-Q. Jiang, L.-S. Liao, Multi-layer pi-stacked molecules as efficient thermally activated delayed fluorescence emitters, Angew. Chem. Int. Edit. 60 (2021) 5213-5219.

[46] S.-Y. Yang, Y.-L. Zhang, F.-C. Kong, Y.-J. Yu, H.-C. Li, S.-N. Zou, A. Khan, Z.-Q. Jiang, L.-S. Liao, Pi-stacked donor-acceptor molecule to realize hybridized local and chargetransfer excited state emission with multi-stimulus response, Chem. Eng. J. 418 (2021) 129366.

[47] M. Hasan, A. Shukla, V. Ahmad, J. Sobus, F. Bencheikh, S.K.M. McGregor, M. Mamada, C. Adachi, S.-C. Lo, E.B. Namdas, Exciton-exciton annihilation in thermally activated delayed fluorescence emitter, Adv. Funct. Mater. 30 (2020) 2000580.

[48] M. Inoue, T. Serevicius, H. Nakanotani, K. Yoshida, T. Matsushima, S. Jursenas, C. Adachi, Effect of reverse intersystem crossing rate to suppress efficiency roll-off in organic lightemitting diodes with thermally activated delayed fluorescence emitters, Chem. Phys. Lett. 644 (2016) 62-67.

[49] G. Kreiza, D. Berenis, D. Banevicius, S. Jursenas, T. Javorskis, E. Orentas, K. Kazlauskas, High efficiency and extremely low roll-off solution- and vacuum-processed oleds based on isophthalonitrile blue TADF emitter, Chem. Eng. J. 412 (2021) 128574.

[50] Z. Qiu, W. Xie, Z. Yang, J.-H. Tan, Z. Yuan, L. Xing, S. Ji, W.-C. Chen, Y. Huo, S.-j. Su, Nanosecond-time-scale delayed fluorescence towards fast triplet-singlet spin conversion for efficient orange-red oleds with negligible efficiency roll-off, Chem. Eng. J. 415 (2021) 128949.

[51] M.H. Cai, M. Auffray, D.D. Zhang, Y.W. Zhang, R. Nagata, Z.S. Lin, X. Tang, C.Y. Chan, Y.T. Lee, T.Y. Huang, X.Z. Song, Y. Tsuchiya, C. Adachi, L. Duan, Enhancing spin-orbital coupling in deep-blue/blue TADF emitters by minimizing the distance from the heteroatoms in donors to acceptors, Chem. Eng. J. 420 (2021) 127591. 